Original Research Paper

\title{
Glutathione S-transferase (T1 \& M1) Null Genotypes as Risk Determinants in Sudanese for Developing Type Two Diabetes Mellitus
}

\author{
${ }^{1}$ Gaafar Mahmoud, ${ }^{2}$ Hisham Mohamed Abdelrahim, \\ ${ }^{3}$ Abed Al Salam Aljahmany and ${ }^{4}$ Eltayeb Mohamed Ahmed Tayrab \\ ${ }^{I}$ Department of Clinical Chemistry, Ribat University Hospital, Khartoum, Sudan \\ ${ }^{2}$ Department of Medicine and Endocrinology, Ribat University Hospital, Khartoum, Sudan \\ ${ }^{3}$ Department of Medical Basic Sciences, Faculty of Applied Medical Sciences, \\ University of Bisha, Bisha, Kingdom of Saudi Arabia \\ ${ }^{4}$ Department of Chemical Pathology, Faculty of Medical Laboratory Sciences, \\ The National Ribat University, Khartoum, Sudan
}

\section{Article history}

Received: 03-06-2020

Revised: 08-09-2020

Accepted: 12-09-2020

Corresponding Author: Eltayeb Mohamed Ahmed Tayrab Department of Chemical Pathology, Faculty of Medical

Laboratory Sciences, The

National Ribat University,

Khartoum, Sudan

Email: eltayebtayrab@gmail.com etayrab@ ribat.edu.sd

\begin{abstract}
Glutathione S-transferases (GSTT1 and GSTM1) are globular proteins responsible for the detoxification of xenobiotics and the prevention of oxidative damage. Functional polymorphisms of the antioxidant glutathione and enzymes have a role in the pathogenicity of T2DM and its complications. The present study is aimed to assess association between GSTM1 and GSTT1 genetic polymorphisms with T2DM in Sudanese people. This case-control study was done in Khartoum. A total of 181 Sudanese (115 T2DM patients and 66 controls) included in this study. Genomic DNA was extracted and processed by Multiplex PCR. The present genotype and null genotypes of GSTT1 and GSTM1 were identified. The biochemical parameters have been estimated in plasma samples. Glycated hemoglobin was also estimated in the whole blood samples. SPSS version 20 was used for the analysis of genotypes distribution, mean values, P-values and Odd Ratio with 95\% confidence intervals. Sudanese patients with T2DM showed an increased frequency of the GSTT1 null genotype when compared to the non-diabetic controls (48.7\% versus $28.8 \%$ ). The diabetic patients showed a lower frequency of GSTT1 genotype compared to their controls $(51.3 \%$ versus $71.2 \%)$. The study revealed a high frequency of the GSTM1 null genotype in diabetic Sudanese compared to their controls (40.9\% versus $36.4 \%$ ); while type-2 diabetes mellitus patients showed a lower frequency of GSTM1 present genotype compared to non-diabetic Sudanese (59.1\% versus 63.6\%). The GSTM1 and GSTTI genes polymorphism can be a good marker for early identification of Sudanese people at risk of T2DM and its complications.
\end{abstract}

Keywords: Glutathione S-transferase (GSTT1), Glutathione S-transferase (GSTM1), Type 2-Diabetes Mellitus, Sudan

\section{Introduction}

Oxidative stress seems to play a significant role in many human diseases. The Glutathione S-transferases (GSTT1 and GSTM1) are globular proteins responsible for the detoxification of xenobiotics and the prevention of oxidative stress (Wang et al., 2006; Amer et al., 2011). Glutathione S-transferases (GSTs), play an essential role in protecting against products of oxidative damage and xenobiotic compounds (Palma-Cano et al., 2017; Wang et al., 2006). The oxidative stress may increase with the polymorphism of GSTM1/GSTT1 genes (Almoshabek et al., 2016; Mustafa et al., 2010). Oxidative stress plays an important role in the pathogenesis of T2DM (Stephens et al., 2009). The polymorphisms of the antioxidant enzymes have been involved in the pathogenesis of T2DM. The low levels of antioxidant enzymes or the non-functionality result in 
excessive reactive metabolites that initiate stress-related pathways thereby leading to insulin resistance and T2DM (Monisha and Vats, 2013). GTTs have an essential role in the detoxification of oxidative stress products, which are one of many risks factors that may be associated with several types of disease affect processes such as T2DM (Sombié et al., 2020). T2DM is growing in a quick manner whole over the world (Raza et al, 2014a; 2014b). Type 2 diabetes is also one of the multifactorial disorders with heterogeneous disturbances of metabolism, the genetics and environmental factors playing important role in its causes (Dadbinpour et al., 2013; Kerner and Brückel, 2014). The cause is either impaired insulin action, insulin secretion or both (Kerner and Brückel., 2014). Diabetes now more than 350 million people worldwide, it is increasing rapidly because of secondary factors, such as obesity, hypertension and lack of physical activity (Cilenšek et al., 2012). In diabetes, due to the defects in cellular metabolism the free radicals increase (Dadbinpour et al., 2013). These radicals react with other vital cellular molecules causing diabetes side effects (Dadbinpour et al., 2013). Sudan is one of the African countries, in which diabetes mellitus is a growing health problem in all socio-economic classes. The national history of the disease is associated with poor glycemic control and a high prevalence of complications (Elrayah et al., 2005). The prevalence of T2DM in Sudan, as in many other low-income countries, is increasing to epidemic proportions, T2DM in Sudan is common among the adult population of northern Sudan (Abdelgadir et al., 2002). The prevalence of T2DM was found to be at range (4-10.8\%) in Northern State and Khartoum State (Elbagir et al., 1996; Osman et al., 2013).

The long term affects and complications of diabetes include progressive development of retinopathy, nephropathy and neuropathy with microvascular and macrovascular disorders. Macrovascular disorders such as atherosclerosis are recognized as major causes of mortality in the diabetic population (Alvin et al., 2001). Although the exact cause of Cardiovascular Diseases (CVD) including atherosclerosis remains unsolved, the pro-oxidative and pro-inflammatory vascular environments are fundamental contributors to the initiation and progression of cardiovascular diseases (Thomas et al., 2008).

This study aimed to find if there any association between genetic polymorphisms of (GSTT1 and GSTM1) in Sudanese with T2DM.

\section{Materials and Methods}

\section{Patients Selection and Data Collection}

In this hospital-based case-control research, which done in Khartoum state and Ribat University Hospital; which is a tertiary care hospital. The period of the study was from September 2015 to May 2018. The study includes 115 Sudanese patients with T2DM (57 females and 58 males) and 66 healthy volunteers as control (29 females 37 and males). The research was approved by the Ethics Research Committee (The National Ribat University). The objectives of the study were explained at the beginning to all individuals under study and written consent was obtained from each participant in the study. The clinical information was collected through questionnaires, by a physician and by the aid of the clinical records.

\section{Biochemical Estimations}

Fasting plasma glucose was measured by the glucose oxidase-peroxidase method. The serum creatinine level was measured by a kinetic Jaffe method. Serum cholesterol was assessed by the cholesterol oxidaseperoxidase method. Serum triglycerides were measured by the glycerol phosphate oxidase-peroxidase amidopyrine method. High-Density Lipoprotein (HDL) cholesterol and Low-Density Lipoprotein (LDL) (Immunoinhibition) were assessed by Mindray BS-380, a fully automated chemical analyzer. Glycated hemoglobin and highly sensitive $\mathrm{C}$ - reactive protein were estimated in full automated chemistry analyzer (COBAS Integra 400 plus).

\section{DNA Extraction}

Peripheral blood (2.5-3.0 mL) was collected from the patients in vacutainers containing EDTA as an anticoagulant. Genomic DNA was extracted in whole blood using the Generation DNA purification capture column kit (Analytica Jena, Berlin Germany) then stored at $-20^{\circ} \mathrm{C}$.

\section{Polymerase Chain Reaction (PCR)}

Primers for GSTT1 and GSTM1 gene were from Microgen Company. GSTT1 primers (GSTT1-Forward: (GAACTCCCTGAAAAGCTAAAGC) and GSTT1Reverse: GTTGGGCTCAAATATACGGTGG) and GSTM1primers (GSTM1-Forward: TTCCTCACTGGTCCTCACATCTC and GSTM1Reverse: TCACCGGATCATGGCCAGCA). Albumin gene primers as internal control, (Albumin-Forward: GCCCTCTGCTAACAAGTCCTAC) (Albumin-Reverse: GCCCTAAAAAG AAAATCGCC AATC) Primers were prepared by using $10 \mu \mathrm{L}$ of primer added to $90 \mu \mathrm{L}$ of sterile de-ionized water. Forward and reverse primers were prepared in separate Eppendorf tubes.

The mixture was prepared by adding $0.25 \mu \mathrm{L}$ of forward primer, $0.25 \mu \mathrm{L}$ of reverse primer of each gene and $17 \mu \mathrm{L}$ sterile water to PCR Premix tube and finally $2 \mu \mathrm{L}$ of DNA. $20 \mu \mathrm{L}$ total volume. The experiment consists of DNA in $0.5 \mathrm{~mL}$ (PCR Premix tube, intron Biotechnology). Using multiplex PCR for all subjects. 
The amplification was performed using initial denaturation at the temperature $95^{\circ} \mathrm{C}$ for $3 \mathrm{~min}$, then followed by 30 cycles at the same temperature $\left(95^{\circ} \mathrm{C}\right)$ for $30 \mathrm{sec}$. Then at $60^{\circ} \mathrm{C}$ for $30 \mathrm{sec}$, followed by $72^{\circ} \mathrm{C}$ for $30 \mathrm{sec}$ and the final extension of $72^{\circ} \mathrm{C}$ for $7 \mathrm{~min}$. The amplified results were identified using electrophoresis in a $1.5 \%$ agarose gel then stained with $0.5 \mathrm{mg} / \mathrm{mL}$ ethidium bromide. The lengths of the product were 480,215 and 350 bp for the GSTT1, GSTM1 and Albumin locus, respectively. The null genotypes and present genotype of GSTM1 and GSTT1 genes were identified.

\section{Results}

The study revealed that; the mean age in the T2DM subjects was $(53.60 \pm 10.08$ years) and in non-diabetic $(51.53 \pm 8.12$ years), respectively. The biochemical and clinical profiles of the patients and the controls were shown in Table 1. The mean Body Mass Index (BMI), Fasting Blood Sugar (FBS), HbA1C, total serum cholesterol, LDL cholesterol and triglycerides in the diabetic patients were significantly higher than in the control volunteers $(\mathrm{P}=0.000)$, but $\mathrm{HDL}$ cholesterol was significantly decreased in diabetic patients $(\mathrm{P}=$ 0.000). Highly sensitive C Reactive Protein (hs-CRP) was significantly increased in diabetic patients compared to their control subjects $(\mathrm{P}=0.007)$. Table 2 shows the comparison of the biochemical parameters according to genotypes; the null genotype of Glutathione S-Transferase enzyme (GSTT1) in diabetes mellitus revealed a significant increase in glucose, hs$\mathrm{CRP}$, total cholesterol and LDL cholesterol ( $\mathrm{P}$ value: $0.004,0.018,0.022 \& 0.002$, respectively) and insignificantly increase in triglycerides and $\mathrm{HbA} 1 \mathrm{C}$, but HDL cholesterol was significantly decreased $(\mathrm{P}=$ 0.021) as shown in (Table2), while the null genotype of glutathione S-transferase enzyme (GSTM1) in diabetes mellitus revealed a significant difference in LDL cholesterol $(\mathrm{P}=0.004)$, (Table 3$)$.

A significant difference in GSTM1\& GSTT1 genotypes distribution in Sudanese patients with T2DM showed a higher frequency of the GSTT1 null genotype compared to their controls $(48.7 \%$ versus $28.8 \%)$ as presented in (Table 4). The diabetic group showed a lower frequency of GSTT1 present genotype compared to their controls $(51.3 \%$ versus $71.2 \%$ ), (OR 2.348, $\mathrm{P}$ value 0.012 ) which reflected a significant association of GSTT1-null genotype with T2DM (Table 4). The study also revealed a higher frequency of the GSTM1 null genotype when compared to their controls $(40.9 \%$ versus $36.4 \%$ ) with (OR: 1.210 and $\mathrm{P}$ : value 0.636 ), as shown in (Table 5).

Table 1: The clinical and biochemical parameters of the Sudanese with type 2 diabetes mellitus patients and their controls

\begin{tabular}{llll}
\hline Parameters & T2DM $(\mathrm{n}=115)$ & Controls $(\mathrm{n}=66)$ & \\
Gender & 58 (males); 57 (females) & 37 (males); 29 (females) & $\mathrm{p} \mathrm{Values}$ \\
\hline Age $($ years) & $53.60 \pm 10.8$ & $51.53 \pm 8.12$ & 0.163 \\
BMI $\left(\mathrm{kg} / \mathrm{m}^{2}\right)$ & $27.76 \pm 3.82$ & $22.939 \pm 1.31$ & $0.000^{* * *}$ \\
FBS $(\mathrm{mg} / \mathrm{dL})$ & $178.13 \pm 77.59$ & $97.74 \pm 8.53$ & $0.000^{* * *}$ \\
Hb A1C $(\%)$ & $8.47 \pm 2.062$ & $4.33 \pm 0.868$ & $0.000^{* * *}$ \\
Cholesterol (mg/dL) & $208.29 \pm 58.878$ & $167.74 \pm 20.256$ & $0.000^{* * *}$ \\
Triglyceride (mg/dL) & $140.96 \pm 60.38$ & $98.95 \pm 27.85$ & $0.000^{* * *}$ \\
LDL (mg/Dl) & $108.97 \pm 37.98$ & $82.52 \pm 11.24$ & $0.000^{* * *}$ \\
HDL (mg/dL) & $44.09 \pm 9.097$ & $49.02 \pm 6.96$ & $0.000^{* * *}$ \\
Highly sensitive C reactive protein (mg//L) & $17.18 \pm 52.12$ & $3.70 \pm 1.75$ & $0.007 * *$ \\
\hline
\end{tabular}

For finding P values; Student's unpaired t-test was used, except in gender comparison. T-test was applied on, (BMI = Body Mass Index; FBS = Fasting Blood Sugar; HDL = High-Density Lipoprotein; LDL = Low-Density Lipoprotein; highly sensitive C-reactive protein). * Stands for significant differences between the groups $(\mathrm{P}<0.05)$

Table 2: Comparison of biochemical parameters in present genotype and null genotype of glutathione transferase enzyme (GSTT1) in Sudanese with type 2 diabetes mellitus

\begin{tabular}{llll}
\hline GSTT1 & Present $(\mathrm{n}=59)$ & Null $(\mathrm{n}=55)$ & P value \\
\hline Glucose $(\mathrm{mg} / \mathrm{dl})$ & $158.56 \pm 67.630$ & $200.05 \pm 82.723$ & $0.004^{* *}$ \\
HbA1c $(\%)$ & $8.141 \pm 2.0587$ & $8.838 \pm 2.0418$ & 0.070 \\
hs CRP $(\mathrm{mg} / \mathrm{l})$ & $5.670 \pm 13.3667$ & $29.830 \pm 72.3284$ & $0.018^{*}$ \\
Total serum cholesterol (mg/dl) & $196.20 \pm 45.425$ & $221.76 \pm 68.868$ & $0.022^{*}$ \\
Serum triglycerides $(\mathrm{mg} / \mathrm{dl})$ & $135.59 \pm 62.175$ & $148.00 \pm 58.045$ & 0.274 \\
HDL $(\mathrm{mg} / \mathrm{dl})$ & $45.99 \pm 7.436$ & $42.06 \pm 10.351$ & $0.021^{*}$ \\
LDL $(\mathrm{mg} / \mathrm{dl})$ & $98.80 \pm 32.747$ & $120.47 \pm 40.317$ & $0.002^{* *}$ \\
BMI $(\mathrm{kg} / \mathrm{m} 2)$ & $28.133 \pm 3.963$ & $27.377 \pm 3.679$ & 0.292 \\
\hline
\end{tabular}

Comparisons were performed by the Student T-test. Data were written as mean \pm SD. $*=$ Stands for significant differences between the groups $(\mathrm{P}<0.05)$ 
Table 3: Comparison of biochemical parameters in present genotype and null genotype of glutathione S-transferase enzyme (GSTM1) in Sudanese with type 2 diabetes mellitus

\begin{tabular}{llll}
\hline GSTMI & Present $(\mathrm{n}=71)$ & Null $(\mathrm{n}=43)$ & P value \\
\hline Glucose & $183.66 \pm 78.375$ & $170.19 \pm 76.994$ & 0.372 \\
HbA1c $(\%)$ & $8.437 \pm 2.1852$ & $8.544 \pm 1.8912$ & 0.789 \\
hs CRP $(\mathrm{mg} / \mathrm{l})$ & $16.530 \pm 57.5736$ & $18.642 \pm 42.907$ & 0.836 \\
Total serum cholesterol (mg/dl) & $210.62 \pm 53.014$ & $205.09 \pm 68.458$ & 0.630 \\
Serum triglycerides $(\mathrm{mg} / \mathrm{dl})$ & $145.52 \pm 53.611$ & $135.07 \pm 70.104$ & 0.372 \\
LDL $(\mathrm{mg} / \mathrm{dl})$ & $116.44 \pm 41.555$ & $97.40 \pm 27.928$ & $0.004 * *$ \\
HDL $(\mathrm{mg} / \mathrm{dl})$ & $43.83 \pm 8.916$ & $44.53 \pm 9.581$ & 0.691 \\
BMI $\left(\mathrm{kg} / \mathrm{m}^{2}\right)$ & $28.026 \pm 3.301$ & $27.328 \pm 4.591$ & 0.386 \\
\hline
\end{tabular}

Comparisons were performed by the Student T-test. Data were written as means \pm SD. ${ }^{*}=$ Stands for significant differences between the groups $(\mathrm{P}<0.05)$

Table 4: Distribution frequencies of genotypes of GSTT1 in Sudanese with T2DM and their control groups and the risk analysis of T2 DM

\begin{tabular}{llllll}
\hline Genotype & T2DM & Control & & & \\
\hline GSTT1 & $\mathrm{n}(\%)$ & $\mathrm{n}(\%)$ & $\mathrm{x} 2$ & OR (95\%CI) & P value \\
Present $(+)$ & $59(51.3 \%)$ & $47(71.2 \%)$ & - & 1 Reference & $0.012 *$ \\
Null $(-)$ & $56(48.7 \%)$ & $19(28.8 \%)$ & 6.849 & $2.348(1.230-4.481)$ & \\
Total & $115(100.0)$ & $66(100.0)$ & & & \\
\hline
\end{tabular}

The comparisons were performed by the chi-square test. *= indicates significant differences between groups $(\mathrm{P}<0.05)$.

Table 5: Distribution frequencies of genotypes of GSTM1 in T2DM and their control groups besides the risk analysis of T2DM

\begin{tabular}{llllll}
\hline Genotype & T2DM & Control & & & \\
\hline GSTM1 & $\mathrm{n}(\%)$ & $\mathrm{n}(\%)$ & $\mathrm{x} 2$ & OR (95\%CI) & P value \\
Present $(+)$ & $68(59.1 \%)$ & $42(63.6 \%)$ & - & 1 Reference & - \\
Null $(-)$ & $47(40.9 \%)$ & $24(36.4 \%)$ & 0.357 & $1.210(0.210-2.258)$ & 0.636 \\
Total & $115(100.0)$ & $66(100.0)$ & & & \\
\hline
\end{tabular}

Comparisons were performed by the chi-square test. $*=$ Stands for significant differences between the groups $(\mathrm{P}<0.05)$

Table 6: Distribution frequencies of genotypes of GSTM1 and risk analysis of T2 DM complications

\begin{tabular}{llllll}
\hline Genotypes & $\begin{array}{l}\text { Present of T2DM } \\
\text { complications }\end{array}$ & $\begin{array}{l}\text { Absent of T2DM } \\
\text { complications }\end{array}$ & & & \\
\hline GSTM1 & $\mathrm{n}(\%)$ & $\mathrm{n}(\%)$ & $(\mathrm{x} 2)$ & OR (95\%CI) & P. value \\
Present $(+)$ & $56(63.6 \%)$ & $12(44.4 \%)$ & 3.149 & $0.457(0.191-1.096)$ & 0.116 \\
Null $(-)$ & $32(36.4 \%)$ & $15(55.6 \%)$ & & & \\
Total & $88(100.0)$ & $27(100.0)$ & & & \\
\hline
\end{tabular}

Comparisons were performed by the chi-square test. $*=$ Stands for significant differences between the groups $(\mathrm{P}<0.05)$.

The GSTT1-null genotype was associated with a 2.348folds increased risk relative to the present genotypes with (P-value 0.012) and the incidence of GSTM1 null was associated with a 1.21 folds increase risk of having T2DM but insignificant ( $\mathrm{P}$ value 0.636 ), while T2DM patients showed a lower frequency of GSTM1 present genotype compared to their controls $(59.1 \% \%$ versus $63.6 \%$ ) as shown in (Table 5). The distribution frequencies of genotypes of GSTM1 and risk analysis of Type 2 DM complications were shown in (Table 6).

\section{Discussion}

Genetic factors play an important role in the pathogenesis of Type-2 Diabetes Mellitus (T2DM) and related complications and that genetically susceptible individuals are likely to develop the disease, when exposed to endogenous and environmental risk factors. Overproduction of Reactive Oxygen Species (ROS) and/or deficiency of antioxidant mechanisms depend on the balance between the generation of ROS and enzymatic or non-enzymatic systems of antioxidative protection, cause an increase of Oxidative Stress (OS). Several factors, such as hypercholesterolemia, glycation of protein, hypertension, diabetes, obesity and aging are risk factors for complications of the diseases, where OS is increased and antioxidant defenses are compromised. Antioxidant enzymes (GSTT1 and GSTM1) represent the second line of defense, which neutralizes lipid peroxidation products as reported by (Sharma et al., 2006). GSTM1 and GSTT1genotypes determine the enzymatic inactivity of the proteins encoded by these genes. The present study suggests that the null genotypes of GSTT1 and GSTM1 are risk factors of developing diabetes mellitus which are consistent with a study done by (Doney et al., 2005); which showed that GSTM1 and GSTT1 null genotypes seem to be genetic risk factors for diseases such as T2DM and its cardiovascular 
complications. The conclusion of this research is also in agreement with a Brazilian study done by (Pinheiro et al., (2013). Many case control studies on GSTM1 and GSTT1 null genotypes had reported a risk associations between diabetes in general and GSTM1 and GSTT1 gene polymorphisms as stated earlier by (Yuille et al., 2002; Chen et al., 2005); while a study done in Romanian population showed that GSTM1 and GSTT1 gene polymorphisms had no associated with the risk of developing T2DM as mentioned by (Stoian et al., 2015).

The frequency of the GSTM1 null genotype in this study is $36.4 \%$; which is lower than that reported in other populations like Egypt $58.62 \%$ by (Nowier et al., 2009), the United Arab Emrates $57.5 \%$ by (Bid et al., 2010) and Northern India 38.61 and $54.00 \%$ by (Raza et al., 2014a). Moreover; (Raza et al., 2014a); suggested that GSTM1 genes deletion can be a predictive marker for early identification of Indians at risk of T2DM. The frequency of the GSTTI null genotype of this study is (48.7\% in T2DM patients) which is lower than what reported in Dubai $(60.0 \%)$ by (Hossaini et al., 2008). On the contrary, the null genotype of GSTTI of Sudanese is higher than Egyptian (35.0\%) (Amer et al., 2011). The genotype frequency of the positive GSTT1 and GSTM1 genes in this study are 71.2 and $63.6 \%$ respectively, which are significantly higher when compared with the Dubai study; where the positive GSTT1 and GSTM1 genes was 40.0 and $42.5 \%$, respectively, as reported by (Hossaini et al., 2008).

\section{Conclusion}

In Sudanese patients with Type-2 Diabetes Mellitus GSTTI null and GSTM1 null genetic polymorphisms are risk factors for the development of diabetes mellitus and GSTTI null is a high risk factor for the development of T2DM complications. These results suggest that GSTT1 and GSTM1 present genotypes cooperatively play a protective role against the development of type 2 diabetes mellitus.

\section{Acknowledgement}

We acknowledge the help of the Internal Medicine (Ribat University Hospital), Ahmed Gasim Fadul Hospital for Cardiac Surgery and Renal Transplant Center, members of molecular laboratory at College of Veterinary Medicine, Sudan University of Science and Technology, Dr. Ehab ELnour Mossaad and Mrs. Suhair Reyhan.

\section{Ethical Approval}

Institutional ethical approval has been obtained to conduct this research study vides project reference number (medical-lab/2015/no.1) dated first September 2015.

\section{Funding}

The authors received no financial support for this study, for the authorship and/or the publication of this paper.

\section{Declaration of Conflict of Interests}

The authors declared that no potential conflicts of interest with respect to this research, authorship and/or the publication of this article.

\section{Authors Contributions}

Gaafar Mahmoud: Prepared the project proposal, collected the samples, did the lab work, did data analysis and wrote the paper.

Hisham Mohamed Abdelrahim: Prepared the project proposal, sample collection, data analysis and wrote the paper.

Abed Al Salam Aljahmany: Shared data analysis, wrote the manuscript and shared the consumables of the research.

Elteyeb Mohammed Ahmed Tayrab: Prepared the research project, shared sample collection, did the data analysis, collected the literature data, wrote the paper, the main supervisor of the research.

\section{References}

Abdelgadir, M., Elbagir, M., Eltom, M., Berne, C., \& Ahr, B. (2002). Reduced leptin concentrations in subjects with type 2 diabetes mellitus in Sudan. MetabolismClinical and Experimental, 51(3), 304-306.

Almoshabek, H. A., Mustafa, M., Al-Asmari, M. M., Alajmi, T. K., \& Al-Asmari, A. K. (2016). Association of glutathione S-transferase GSTM1 and GSTT1 deletion polymorphisms with obesity and their relationship with body mass index, lipoprotein and hypertension among young age Saudis. JRSM cardiovascular disease, 5, 2048004016669645.

Alvin, C. P., Bernrdwald, E., Fauce, A.S., Kasper, D. L., Hveser, S. L., Longo, D. L., Jambon, J. L., \& Heinson (eds) (2001). Diabetes in Principle of internal medicine. U.S.A. New York, Megronhill,

Amer, M. A., Ghattas, M. H., Abo-Elmatty, D. M., \& Abou-El-Ela, S. H. (2011). Influence of glutathione S-transferase polymorphisms on type- 2 diabetes mellitus risk. Genet Mol Res, 10(4), 3722-3730.

Bid, H. K., Konwar, R., Saxena, M., Chaudhari, P., Agrawal, C. G., \& Banerjee, M. (2010). Association of glutathione S-transferase (GSTM1, T1 and P1) gene polymorphisms with type 2 diabetes mellitus in north Indian population. Journal of postgraduate medicine, 56(3), 176.

Chen, K., Jiang, Q. T., \& He, H. Q. (2005). Relationship between metabolic enzyme polymorphism and colorectal cancer. World journal of gastroenterology: WJG, 11(3), 331. 
Cilenšek, I., Mankoč, S., Petrovič, M. G., \& Petrovič, D. (2012). GSTT1 null genotype is a risk factor for diabetic retinopathy in Caucasians with type 2 diabetes, whereas GSTM1 null genotype might confer protection against retinopathy. Disease markers, 32(2), 93-99.

Dadbinpour, A., Sheikhha, M. H., Darbouy, M., \& Afkhami-Ardekani, M. (2013). Investigating GSTT1 and GSTM1 null genotype as the risk factor of diabetes type 2 retinopathy. Journal of Diabetes \& Metabolic Disorders, 12(1), 48.

Doney, A. S., Lee, S., Leese, G. P., Morris, A. D., \& Palmer, C. N. (2005). Increased cardiovascular morbidity and mortality in type 2 diabetes is associated with the glutathione S-transferase thetanull genotype: A Go-DARTS study. Circulation, 111(22), 2927-2934.

Elbagir, M. N., Eltom, M. A., Elmahadi, E. M., Kadam, I. M., \& Berne, C. (1996). A population-based study of the prevalence of diabetes and impaired glucose tolerance in adults in northern Sudan. Diabetes care, 19(10), 1126-1128.

Elrayah, H., Eltom, M., Bedri, A., Belal, A., Rosling, H., \& Östenson, C. G. (2005). Economic burden on families of childhood type 1 diabetes in urban Sudan. Diabetes research and clinical practice, 70(2), 159-165.

Hossaini, A. M., Zamrroni, I. M., Kashem, R. A., \& Khan, Z. F. (2008). Polymorphism of glutathione Stransferases as genetic risk factors for the development of complications in type 2 diabetes mellitus. Journal of Critical Care, 23(3), 444-448.

Kerner, W., \& Brückel, J. (2014). Definition, classification and diagnosis of diabetes mellitus. Experimental and clinical endocrinology \& diabetes, 122(07), 384-386.

Monisha, B. \& Vats, P. (2013). Reactive metabolites and antioxidant gene polymorphisms in type 2 diabetes mellitus. Redox biology, 2, 170-177.

Mustafa, M. D., Pathak, R., Ahmed, T., Ahmed, R. S., Tripathi, A. K., Guleria, K., \& Banerjee, B. D. (2010). Association of glutathione S-transferase M1 and $\mathrm{T} 1$ gene polymorphisms and oxidative stress markers in preterm labor. Clinical biochemistry, 43(13-14), 1124-1128.

Nowier, S. R., Kashmiry, N. K., Rasool, H. A. A., Morad, H., \& Ismail, S. (2009). Association of Type 2 diabetes mellitus and glutathione S-transferase (GSTM1 and GSTT1) genetic polymorphism. Res J Med Medical Sci, 4(2), 181-188.

Osman, H. A., Elsadek, N., \& Abdullah, M. A. (2013). Type 2 diabetes in Sudanese children and adolescents. Sudanese journal of paediatrics, 13(2), 17.

Palma-Cano, L. E., Córdova, E. J., Orozco, L., MartínezHernández, A., Cid, M., Leal-Berumen, I., ... \& Moreno-Brito, V. (2017). GSTT1 and GSTM1 null variants in Mestizo and Amerindian populations from northwestern Mexico and a literature review. Genetics and molecular biology, 40(4), 727-735.
Pinheiro, D. S., Rocha Filho, C. R., Mundim, C. A., de Marco Júnior, P., Ulhoa, C. J., Reis, A. A., \& Ghedini, P. C. (2013). Evaluation of glutathione S-transferase GSTM1 and GSTT1 deletion polymorphisms on type2 diabetes mellitus risk. PLOS One, 8(10), e76262.

Raza, S. T., Abbas, S., Ahmad, A., Ahmed, F., Zaidi, Z. H., \& Mahdi, F. (2014a). Association of glutathioneS-transferase (GSTM1 and GSTT1) and FTO gene polymorphisms with type 2 diabetes mellitus cases in Northern India. Balkan Journal of Medical Genetics, 17(1), 47-54. (a)

Raza, S. T., Fatima, J., Ahmed, F., Abbas, S., Zaidi, Z. H., Singh, S., \& Mahdi, F. (2014b). Association of angiotensin-converting enzyme (ACE) and fatty acid binding protein 2 (FABP2) genes polymorphism with type 2 diabetes mellitus in Northern India. Journal of the Renin-AngiotensinAldosterone System, 15(4), 572-579. (b)

Sharma, R., Ansari, G.A.S., \& Awasthi, Y.C. (2006). Physiological substrates of Glutathione S-Transferases. In: Awasthi, Y.C. (ed) Toxicology of Glutation Transferases, (pp. 179-203). CRC Press, New York.

Sombié, H. K., Sorgho, A. P., Kologo, J. K., Ouattara, A. K., Yaméogo, S., Yonli, A. T., ... \& Bado, P. (2020). Glutathione S-transferase M1 and T1 genes deletion polymorphisms and risk of developing essential hypertension: a case-control study in Burkina Faso population (West Africa). BMC medical genetics, 21(1), 1-10.

Stephens, J. W., Khanolkar, M. P., \& Bain, S. C. (2009). The biological relevance and measurement of plasma markers of oxidative stress in diabetes and cardiovascular disease. Atherosclerosis, 202(2), 321-329.

Stoian, A., Bănescu, C., Bălaşa, R. I., Moţăţăianu, A., Stoian, M., Moldovan, V. G., ... \& Dobreanu, M. (2015). Influence of GSTM1, GSTT1 and GSTP1 polymorphisms on type 2 diabetes mellitus and diabetic sensorimotor peripheral neuropathy risk. Disease markers, 2015.

Thomas, S. R., Witting, P. K., \& Drummond, G. R. (2008). Redox control of endothelial function and dysfunction: molecular mechanisms and therapeutic opportunities. Antioxidants \& redox signaling, 10(10), 1713-1766.

Wang, G., Zhang, L., \& Li, Q. (2006). Genetic polymorphisms of GSTT1, GSTM1 and NQO1 genes and diabetes mellitus risk in Chinese population. Biochemical and biophysical research communications, 341(2), 310-313.

Yuille, M., Condie, A., Hudson, C., Kote-Jarai, Z., Stone, E., Eeles, R., .. \& Houlston, R. (2002). Relationship between glutathione S-transferase M1, $\mathrm{T} 1$ and P1 polymorphisms and chronic lymphocytic leukemia. Blood, The Journal of the American Society of Hematology, 99(11), 4216-4218. 